\title{
Innovación docente en la aplicación de un ciclo de mejora en el aula para el alumnado de la asignatura de Fundamentos de Podología
}

\section{Teaching innovation in the application of a cycle of improvement in the classroom for students of the subject of Podiatry Fundamentals}

MANUEl COHEÑA JimÉnez

ORCID: https://orcid.org/0000-0001-5714-6594

Universidad de Sevilla.

Departamento de Podología

mcohena@us.es

Fecha de recepción: 18/11/2019

Fecha de aceptación: 24/11/2019

DOI: http://dx.doi.org/10.12795/9788447221912.090

Pp.: 2029-2052 


\section{Resumen}

Este artículo tiene como objetivo realizar una mejora e innovación docente en la asignatura Fundamentos de Podología de 1o Curso de Grado en Podología. A través de una metodología de aprendizaje de los contenidos teóricos con actividades y evaluaciones mediante escaleras de aprendizaje. El objetivo fundamental es trabajar de manera individual y colectiva los contenidos conceptuales, históricos, legales y profesionales que constituyen la podología fundamental. Es el aprendizaje de la Podología básica para la formación de futuros profesionales de la Podología basado en el conocimiento y la evidencia científica. Los resultados de este ciclo de mejora en el aula muestran eficacia en el proceso de enseñanza y aprendizaje de los contenidos.

Palabras clave: Fundamentos de Podología, Grado en Podología, docencia universitaria, experimentación docente universitaria.

\section{Abstract}

This article aims to make a teaching improvement and innovation in the subject Podiatry Fundamentals of first Degree Course in Podiatry. Through a methodology for learning theoretical contents with activities and assessments through learning ladders. The main objective is to work individually and collectively the conceptual, historical, legal and professional contents that constitute the fundamental podiatry. It is the learning of basic podiatry for the training of future podiatry professionals based on knowledge and scientific evidence. The results of this cycle of improvement in the classroom show effectiveness in the process of teaching and learning the contents.

Key words: Podiatry Fundamentals, Podiatry Degree, university teaching, university teaching experimentation.

Jornadas de Formación e Innovación Docente del Profesorado | № 2 (2019) Esta obra se distribuye con la licencia Creative Commons 


\section{Descripción del contexto}

La titulación de Grado de Podología de la Facultad de Enfermería, Fisioterapia y Podología de la Universidad de Sevilla (US) contiene una asignatura obligatoria de 6 créditos ECTS en su primer curso, denominada "Fundamentos de Podología". Se desarrolla durante el primer cuatrimestre y va a ser objeto del presente trabajo mediante la aplicación de un ciclo de mejora en el aula (CIMA) como ampliación y con mejoras a la realizada en el curso anterior, durante el año 2018. La temática abordada se centra en contenidos conceptuales, profesionales e históricos de la titulación, trabajando competencias transversales que el estudiante demostrará a lo largo de sus estudios universitarios y posteriormente en su vida profesional. La asignatura se imparte en un solo grupo, excepto algunos seminarios que se componen de 4 grupos. Toda la clase participará en el CIMA, debido a que se trata de un grupo único, con una matriculación de 61 estudiantes. Esta asignatura de primer curso pretende que el alumno adquiera unas herramientas y conceptos que les van a ser requeridos en su futuro como podólogos. La media de edad se sitúa entre 18 y 20 años, lo que implica una cierta dificultad de manejo de grupo. Están por primera vez en el sistema educativo universitario y necesitan adaptarse, por lo que reconozco que haberlo hecho atractivo para su aprendizaje ha sido una complicada tarea pero a pesar de ello reconozco que los resultados obtenidos fueron positivos.

Con respecto a la descripción física de las aulas disponibles en el Centro Docente de Fisioterapia y Podología de la Universidad de Sevilla, sus aulas presentan una distribución clásica y rígida con las mesas y sillas fijadas al suelo y solamente en alguna ocasión se podrá hacer uso de espacios con mesas y sillas móviles, para los grupos reducidos, que permitieron una mejor proceso de comunicación entre el alumnado favoreciendo el proceso de enseñanza-aprendizaje. La mayoría de las actividades se 
desarrollaran en espacios con una bancada de sillas juntas y con una capacidad de 9 estudiantes por fila. Para completar el aula dispone de una mesa sobre una tarima elevada con un ordenador para el aula y una pizarra manual y otra digital. Este CIMA se desarrollará en el bloque temático de "Fundamentos de Podología", concretamente en las Unidades Temáticas II sobre el Marco Histórico, en la Unidad Temática III sobre el Marco Conceptual, y en la Unidad Temática V sobre el Marco Profesional. Cada parte de las Unidades temáticas que integraron este CIMA componen un total de 20 horas que es la duración de este CIMA, desde el 24/09/19 hasta el 15/11/19.

\section{Diseño previo del CIMA}

\section{Mapa de contenidos y modificaciones introducidas en el CIMA del curso 2019/2020}

Un mapa de contenidos es más que un mapa conceptual, que incluye procedimientos, valores y actitudes que permite conectar los pensamientos y localizarlos de manera visual y además te permite una priorización de los contenidos. Se ha realizado una selección de los contenidos para la elaboración y diseño del mapa de contenidos. Siendo fundamental tener en cuenta los tiempos, el programa, criterios de evaluación y eligiendo entre la calidad y cantidad de los contenidos que se deben relacionar entre sí. La esencia de estos se basa en la formulación de un problema central que se resuelve con otros sub-problemas y cuyos contenidos conceptuales presentan contenidos jerarquizantes y estructurantes. Es fundamental crear un entorno para el aprendizaje crítico natural (Bain, 2007) diseñando preguntas intrigantes y trabajando con ideas previas, teniendo en cuenta lo que piensan nuestros estudiantes. Y así conocer el esquema mental de los 
estudiantes para iniciar el proceso de motivación y el aprendizaje de éstos. En este CIMA se han mejorado los mapas de contenidos y ampliado un poco más con el trabajo realizado por el alumnado, para favorecer el proceso de enseñanza-aprendizaje y como mejora propuesta a partir del ciclo de mejora realizado en el año anterior (Coheña, 2018). Está comprobado que mediante la creación de un problema, un proyecto o resolución de un caso clínico en relación a los contenidos se puede captar la atención del alumnado en la búsqueda de esos problemas y su resolución, además de fomentar su participación en la construcción del conocimiento. Este aspecto se potencia con el trabajo en subgrupos y con un aprendizaje colaborativo y experiencial que fomente la inteligencia y el aprendizaje colectivos. Para ello se han elaborado los siguientes mapas de contenidos con contenido actitudinal (círculo amarillo), procedimental (triangulo rosa) y conceptual (círculos negro). Presentando 3 mapas de contenidos (Figuras 1, 2 y 3).

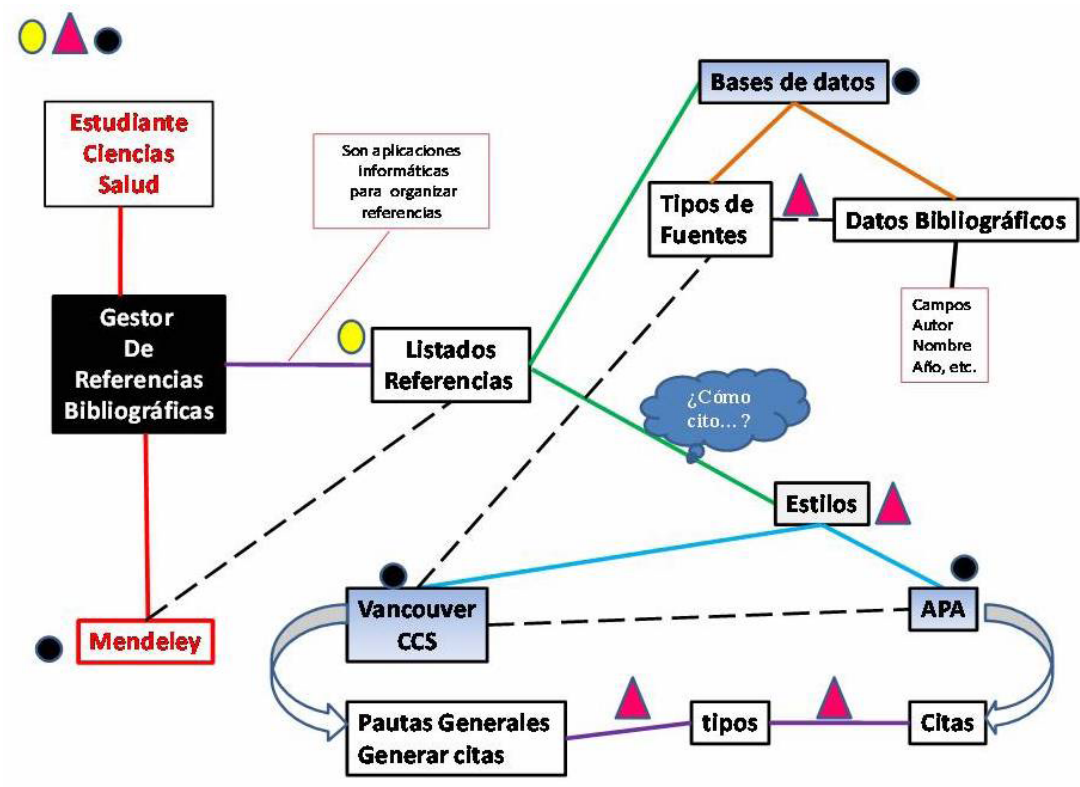

Figura 1. Mapa de contenidos sobre el gestor de referencias y estilos bibliográficos.

Jornadas de Formación e Innovación Docente del Profesorado | № 2 (2019) Esta obra se distribuye con la licencia Creative Commons Reconocimiento-NoComercial-SinObraDerivada Internacional (CC BY-NC-ND 4.0.) 


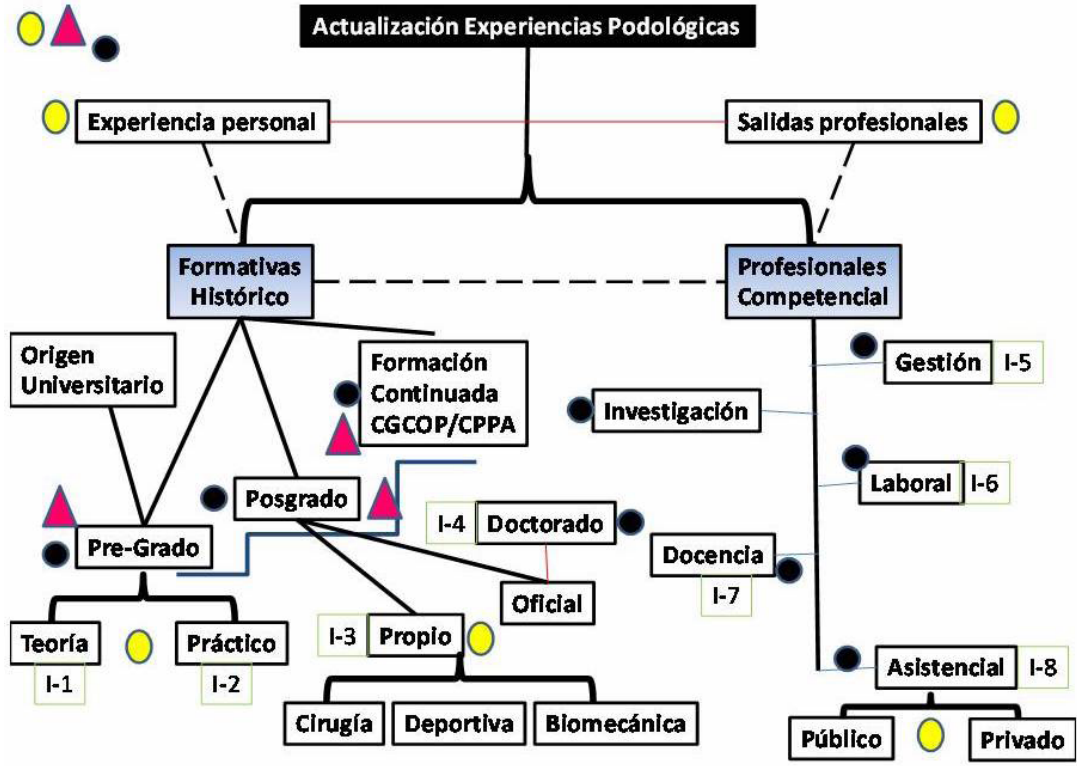

Figura 2. Mapa de contenidos sobre salidas y experiencias profesionales de Podología.

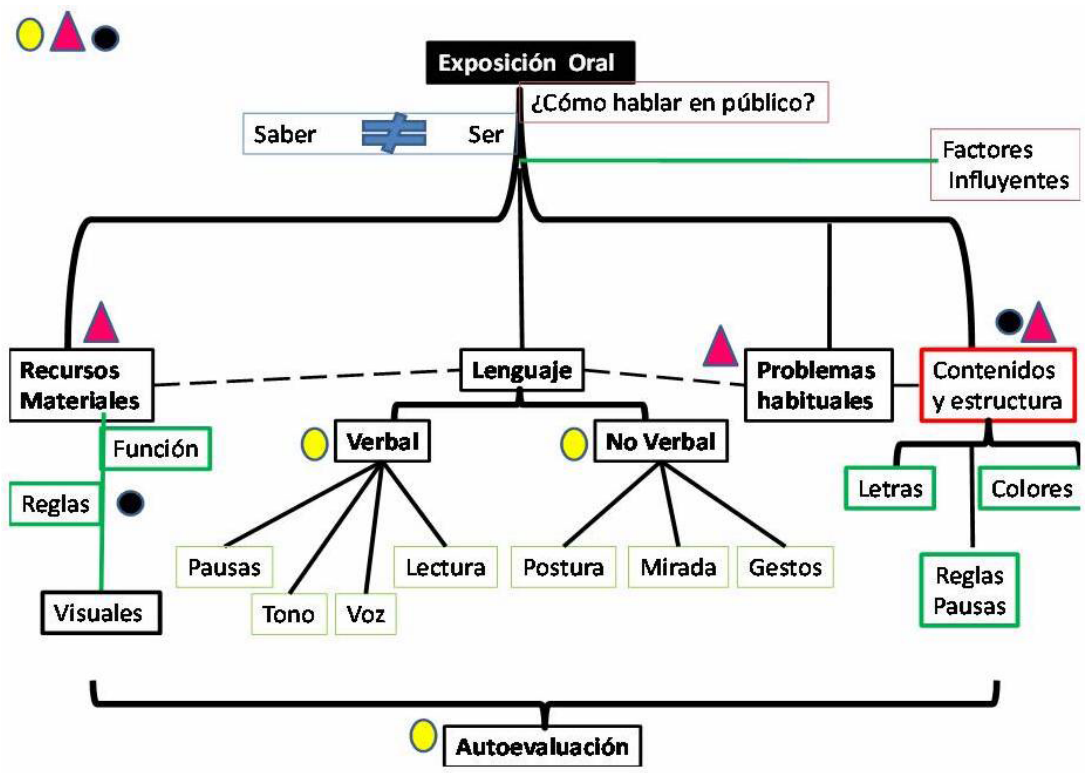

Figura 3.Mapa de contenidos sobre la comunicación en entrevista clínica.

Jornadas de Formación e Innovación Docente del Profesorado | № 2 (2019) Esta obra se distribuye con la licencia Creative Commons Reconocimiento-NoComercial-SinObraDerivada Internacional (CC BY-NC-ND 4.0.) 
Este CIMA integra contenidos de enseñanza: conceptuales, procedimentales y actitudinales que se trabajan en clase y que junto con los mapas de contenidos y problemas describen el aprendizaje que se pretende conseguir. En el temario de la asignatura consta como contenido conceptual: Unidad Temática II: Marco Histórico. Experiencias sobre Podología actual. Comunicación de experiencias formativas y profesionales. Unidad Temática III: Marco Conceptual. Fuentes de documentación en Podología. Lectura, interpretación y comunicación de documentos científicos. Unidad Temática V: Marco Profesional. Funciones y Organizaciones profesionales en Podología. Comunicación en Podología. Principios básicos de la Entrevista clínica podológica. Tecnologías de la Información y la Comunicación aplicadas a Podología. Aplicación de las nuevas tecnologías de la información y la comunicación al campo de la Podología. Biblioteca. Búsqueda bibliográfica. Preparación de documentos con las nuevas herramientas informáticas. Las diferentes fuentes de documentación y bases de datos específicas de Ciencias de la salud, junto a los gestores bibliográficos son objeto de análisis en cuanto a los contenidos de tipo procedimental. Se pretende fomentar la inteligencia colectiva mediante un aprendizaje basado en la experiencia y reflexiones de los estudiantes y la realización de trabajo autónomo y colaborativo (Guerra-Martín, Lima-Serrano, Zambrano-Domínguez y Fernández-Rodríguez, 2013; Guerra-Martín, Lima-Serrano, Zambrano-Domínguez y Lima-Rodríguez, 2014).

En este CIMA se trabaja otro pilar importante en el proceso de aprendizaje y que son los contenidos actitudinales, debido a que la titulación de Podología no es de primera elección para el alumnado matriculado. Un porcentaje importante de nuestros estudiantes tiene como primera elección otras titulaciones como Fisioterapia, Odontología o Medicina. Por este motivo entiendo que es fundamental generar en ellos sentimientos de entusiasmo, interés y motivación. Uno de los objetivos de este CIMA es la 
participación activa del alumnado que es el protagonista del proceso de enseñanza-aprendizaje, promoviendo las búsquedas de información autónomas (Guerra-Martín et al., 2013; Guerra-Martín et al., 2014) y siguiendo el método científico. Si bien he de reconocer que algunos de los estudiantes se sorprenden de ser ellos la parte activa del proceso de enseñanza. En mi opinión, los profesional de la salud y por tanto los futuros profesionales en Podología guiaran sus actuaciones basándose y fundamentándose en el método científico y en la Podología basada en la evidencia.

\section{Modelo metodológico posible}

Antes de implantar esta nueva metodología en la asignatura, se empleaba un desarrollo tradicional basado en el control de asistencia a clase y que definía mi modelo metodológico real. Todo esto se modificó en el curso 2018/2019 cuando realicé la primera experiencia como Ciclo de Mejor Docente y utilicé los mapas de contenidos y una evaluación mediante el uso de escaleras de aprendizaje (Coheña, 2018). Los resultados mostraron, en general, una adquisición adecuada de los conocimientos trabajados y fueron satisfactorios para el alumnado y el profesorado. En relación a la descripción de mis clases, he de decir que casi siempre se impartía la clase como no magistral pura, para ello abordo los contenidos y se puede participar en todo momento, acto que fomento. Planteo problemas, o incluso temas de actualidad y resuelvo dudas. Durante la clase no suelo estar en el atril, incluso ni cerca de la pizarra y/o tarima. Por todo ello, mi modelo metodológico ideales realizar una metodología de trabajo colaborativa en clase. No todo el alumnado realiza el trabajo autónomo en casa (Tourón y Santiago, 2015). En consecuencia, el modelo metodológico posible comienza con una introducción y resumen previo en clase de lo tratado en clases anteriores y resolviendo dudas. Éste se 
representa en la Figura 4 , donde $\mathrm{R}=$ Breve recordatorio teórico; $T=$ Teoría; $D=$ Debate y reflexiones sobre el tema; $P=$ Práctica desarrollada por el alumnado; $\mathrm{C}=$ Conclusiones y

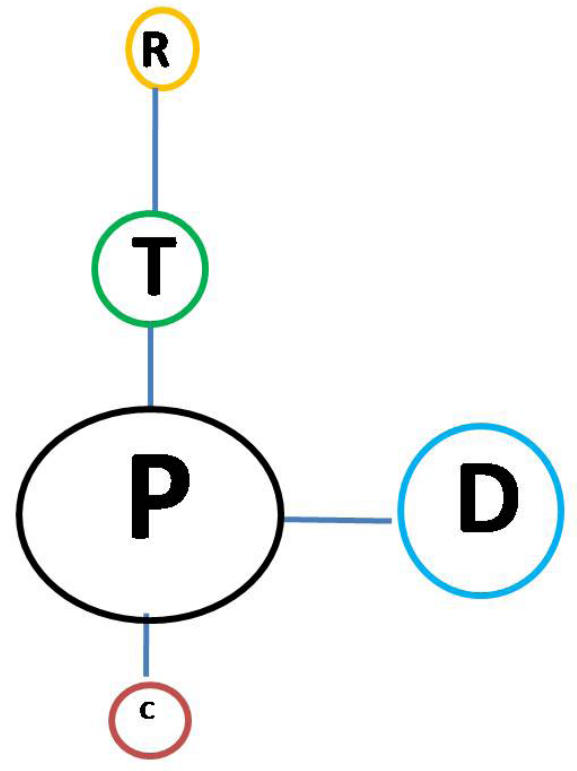

Figura 4. Modelo metodológico posible.

Al realizar el CIMA con un grupo numeroso una de las mejoras propuestas con respecto a la metodología empleada, fue crear grupos de trabajo más reducidos, pero a pesar de ello se generaran muchos subgrupos. De manera que cada subgrupo estaba integrado por 5 estudiantes. No obstante la descripción fisico-estructural del aula fue un problema para el desarrollo normal de la actividad, con aulas de mesas y sillas fijadas al suelo. Mi papel en estas clases fue de guía aclarando dudas y conduciendo el discurso de opiniones o discrepancias (Finkel, 2000). El diario profesional permite realizar reflexiones sobre si es mejor el trabajo en tantos sub-grupos o no. Hoy en día, sigo con esas reflexiones en mi cabeza. El modelo metodológico se basaría en plantear la pregunta inicial, seguido de un trabajo colaborativo con búsqueda de información autónoma de los sub-grupos, que plantearan 
cuestiones de los tema y finalmente se valorará el proceso de enseñanza. El aprendizaje activo de los estudiantes mediante una dinámica cooperativa mejora la relación interpersonal, contando con las opiniones de todos, motivando el aprendizaje de contenidos, y mejorando las habilidades comunicativas. La atención personalizada es un eje prioritario para conseguir el mejor rendimiento de los estudiantes.

\section{Secuencia de actividades programada}

Se diseñó una secuencia de actividades (Tabla 1, 2, 3 y 4) para la realización de este CIMA.

Tabla 1 Secuencia de actividades centrado en los gestores bibliográficos.

\begin{tabular}{|c|c|c|}
\hline Tipo de Actividad & Descripción & Tiempo \\
\hline $\begin{array}{l}\text { Actividad de presentación } \\
\text { de actividad y } \\
\text { planteamiento de dudas }\end{array}$ & $\begin{array}{l}\text { Se presenta contenido, metodología y } \\
\text { evaluación y se resuelven dudas }\end{array}$ & $10^{\prime}$ \\
\hline $\begin{array}{l}\text { Actividad de cuestionario } \\
\text { inicial de conocimientos }\end{array}$ & $\begin{array}{l}\text { Recogida de ideas previas del } \\
\text { alumnado }\end{array}$ & $15^{\prime}$ \\
\hline $\begin{array}{l}\text { Actividad de planteamiento } \\
\text { del problema }\end{array}$ & $\begin{array}{l}\text { Se plantean los problemas: ¿Conoces } \\
\text { algún gestor de referencias } \\
\text { bibliográficas? ¿Cuál es su utilidad? Se } \\
\text { debate y resuelven de dudas }\end{array}$ & $20^{\prime}$ \\
\hline $\begin{array}{l}\text { Actividad de taller } \\
\text { específico sobre el gestor }\end{array}$ & $\begin{array}{l}\text { Se trabajan los contenidos específicos } \\
\text { del gestor }\end{array}$ & $60^{\prime}$ \\
\hline $\begin{array}{l}\text { Actividad de trabajo } \\
\text { colaborativo }\end{array}$ & $\begin{array}{l}\text { Se trabaja en equipo en grupos de 3-4 } \\
\text { estudiantes empleando la herramienta }\end{array}$ & $40^{\prime}$ \\
\hline Actividad de resolución & Puesta en común y resolución de dudas & $20^{\prime}$ \\
\hline $\begin{array}{l}\text { Actividad de conclusiones } \\
\text { finales }\end{array}$ & $\begin{array}{l}\text { Se realiza una sintesis y conclusiones } \\
\text { finales de ideas }\end{array}$ & $10^{\prime}$ \\
\hline $\begin{array}{l}\text { Actividad de Cuestionario } \\
\text { final sobre la actividad y } \\
\text { los conocimientos }\end{array}$ & $\begin{array}{l}\text { Recogida de ideas finales aprendidas } \\
\text { por el alumnado }\end{array}$ & $20^{\prime}$ \\
\hline
\end{tabular}

Jornadas de Formación e Innovación Docente del Profesorado I № 2 (2019) Esta obra se distribuye con la licencia Creative Commons Reconocimiento-NoComercial-SinObraDerivada Internacional (CC BY-NC-ND 4.0.) 
Tabla 2. Secuencia de actividades de contenidos de referencias bibliográficas para la elaboración de documentos científicos.

\begin{tabular}{|c|c|c|}
\hline Tipo de Actividad & Descripción & Tiempo \\
\hline $\begin{array}{l}\text { Actividad de presentación } \\
\text { de la actividad y } \\
\text { planteamiento de dudas }\end{array}$ & $\begin{array}{l}\text { Se presenta contenido, } \\
\text { metodología y evaluación y se } \\
\text { resuelven dudas }\end{array}$ & $5^{\prime}$ \\
\hline $\begin{array}{l}\text { Actividad de Cuestionario } \\
\text { inicial de conocimientos }\end{array}$ & $\begin{array}{l}\text { Recogida de ideas previas del } \\
\text { alumnado }\end{array}$ & $15^{\prime}$ \\
\hline $\begin{array}{l}\text { Actividad de Planteamiento } \\
\text { del problema }\end{array}$ & $\begin{array}{l}\text { Se plantean preguntas clave: } \\
\text { ¿Conoces los estilos APA y } \\
\text { Vancouver de referencias } \\
\text { bibliográficas? Se solicita } \\
\text { opiniones, se debate y plantean } \\
\text { dudas }\end{array}$ & $20^{\prime}$ \\
\hline $\begin{array}{l}\text { Actividad de formación de } \\
\text { grupos de trabajo }\end{array}$ & $\begin{array}{l}\text { Se componen los grupos } \\
\text { iniciales de trabajo y se entrega } \\
\text { materiales por el profesorado }\end{array}$ & $15^{\prime}$ \\
\hline $\begin{array}{l}\text { Actividad de trabajo } \\
\text { colaborativo en grupos } \\
\text { formados }\end{array}$ & $\begin{array}{l}\text { Trabajo en grupos } 1 \text { y } 2 . \\
\text { Asignación de contenidos a } \\
\text { trabajar los estudiantes (Libros, } \\
\text { Cap de libros, revistas, etc..) y } \\
\text { elije estudiante representante } \\
\text { final. }\end{array}$ & $70^{\prime}$ \\
\hline $\begin{array}{l}\text { Actividad de Creación } \\
\text { del grupo de estudiantes } \\
\text { expertos. }\end{array}$ & $\begin{array}{l}\text { Se crea el grupo de estudiantes } \\
\text { expertos para exponer a toda la } \\
\text { clase. Trabajo en grupo. }\end{array}$ & $15^{\prime}$ \\
\hline $\begin{array}{l}\text { Actividad de Exposición } \\
\text { final. }\end{array}$ & $\begin{array}{l}\text { El grupo experto expone a la } \\
\text { clase completa de estudiantes. } \\
\text { Se debate y plantean y } \\
\text { resuelven dudas }\end{array}$ & $30^{\prime}$ \\
\hline $\begin{array}{l}\text { Actividad de Cuestionario } \\
\text { final sobre la actividad y los } \\
\text { conocimientos }\end{array}$ & $\begin{array}{l}\text { Recogida de ideas finales } \\
\text { aprendidas por el alumnado }\end{array}$ & $20^{\prime}$ \\
\hline
\end{tabular}

Jornadas de Formación e Innovación Docente del Profesorado | № 2 (2019) Esta obra se distribuye con la licencia Creative Commons Reconocimiento-NoComercial-SinObraDerivada Internacional (CC BY-NC-ND 4.0.) 
Tabla 3. Secuencia de actividades centrado en la motivación personal, como estudiantes y profesionales. Análisis de las salidas profesionales de la titulación.

\begin{tabular}{|c|c|c|}
\hline Tipo de Actividad & Descripción & Tiempo \\
\hline $\begin{array}{l}\text { Actividad de Presentación } \\
\text { de la primera actividad }\end{array}$ & $\begin{array}{l}\text { Se presenta contenido, } \\
\text { metodología y evaluación y se } \\
\text { resuelven dudas }\end{array}$ & $10^{\prime}$ \\
\hline $\begin{array}{l}\text { Actividad de motivación } \\
\text { personal y profesional }\end{array}$ & $\begin{array}{l}\text { Video-forum: El circo de } \\
\text { las mariposas, fomentando } \\
\text { motivación y análisis en el } \\
\text { futuro profesional }\end{array}$ & $50^{\prime}$ \\
\hline Actividad de Debate & $\begin{array}{l}\text { Debate y opiniones en } \\
\text { torno a la motivación y a la } \\
\text { construcción del conocimiento } \\
\text { de la misma }\end{array}$ & $20^{\prime}$ \\
\hline $\begin{array}{l}\text { Actividad de Presentación } \\
\text { de la segunda actividad }\end{array}$ & $\begin{array}{l}\text { Se presenta el contenido, } \\
\text { metodología y evaluación }\end{array}$ & $10^{\prime}$ \\
\hline $\begin{array}{l}\text { Actividad de Cuestionario } \\
\text { inicial de conocimientos }\end{array}$ & $\begin{array}{l}\text { Recogida de ideas previas del } \\
\text { alumnado }\end{array}$ & $20^{\prime}$ \\
\hline $\begin{array}{l}\text { Actividad de Presentación } \\
\text { de Invitados }\end{array}$ & $\begin{array}{l}\text { Debate y experiencias formativa } \\
\text { y profesional de los invitados. } \\
\text { Se aborda las experiencias } \\
\text { antes, durante y después de } \\
\text { los estudios y siguiendo pautas } \\
\text { asignadas por el profesorado. } \\
\text { Visibiliza especialidades y } \\
\text { competencias profesionales } \\
\text { propias de la titulación. }\end{array}$ & $35^{\prime}$ \\
\hline $\begin{array}{l}\text { Actividad de Participación } \\
\text { experiencial profesional. }\end{array}$ & $\begin{array}{l}\text { Participación experiencial } \\
\text { mediante un debate público y } \\
\text { formativo de profesionalización. }\end{array}$ & $180^{\prime}$ \\
\hline $\begin{array}{l}\text { Actividad de Planteamiento } \\
\text { de dudas y problemas }\end{array}$ & $\begin{array}{l}\text { Se plantean dudas y resuelven } \\
\text { problemas relacionados con las } \\
\text { salidas profesionales. }\end{array}$ & $50^{\prime}$ \\
\hline $\begin{array}{l}\text { Actividad de Cuestionario } \\
\text { final de los conocimientos }\end{array}$ & $\begin{array}{l}\text { Recogida de ideas finales } \\
\text { aprendidas por el alumnado }\end{array}$ & $25^{\prime}$ \\
\hline
\end{tabular}

Jornadas de Formación e Innovación Docente del Profesorado | № 2 (2019) Esta obra se distribuye con la licencia Creative Commons Reconocimiento-NoComercial-SinObraDerivada Internacional (CC BY-NC-ND 4.0.) 
Tabla 4. Secuencia de actividades del ciclo de mejora centrado en la comunicación en entrevista clínica, comunicación oral y expresión como profesionales de la salud.

\begin{tabular}{|c|c|c|}
\hline Tipo de Actividad & Descripción & Tiempo \\
\hline $\begin{array}{l}\text { Actividad de presentación } \\
\text { de la actividad }\end{array}$ & $\begin{array}{l}\text { Se presenta contenido, } \\
\text { metodología y evaluación y se } \\
\text { resuelven dudas }\end{array}$ & $5^{\prime}$ \\
\hline $\begin{array}{l}\text { Actividad de cuestionario } \\
\text { inicial de conocimientos }\end{array}$ & $\begin{array}{l}\text { Recogida de ideas previas del } \\
\text { alumnado }\end{array}$ & $20^{\prime}$ \\
\hline $\begin{array}{l}\text { Actividad de } \\
\text { conocimientos sobre } \\
\text { comunicación oral }\end{array}$ & $\begin{array}{l}\text { Se comienza: "lluvia de ideas" en } \\
\text { relación a la comunicación oral. } \\
\text { Debate y planteamientos }\end{array}$ & $20^{\prime}$ \\
\hline $\begin{array}{l}\text { Actividad de formación de } \\
\text { grupos de trabajo }\end{array}$ & $\begin{array}{l}\text { Formación de grupos de trabajo: } \\
\text { "ideal: con el profesorado" y sub- } \\
\text { grupos. Reparto de contenidos }\end{array}$ & $10^{\prime}$ \\
\hline $\begin{array}{l}\text { Actividad de trabajo } \\
\text { autónomo y colaborativo }\end{array}$ & $\begin{array}{l}\text { Trabajo autónomo y colaborativo } \\
\text { de los grupos en función del } \\
\text { contenido asignado }\end{array}$ & $40^{\prime}$ \\
\hline $\begin{array}{l}\text { Actividad de trabajo del } \\
\text { grupo "ideal" }\end{array}$ & $\begin{array}{l}\text { Trabajo de los grupos con el } \\
\text { miembro del grupo "ideal" }\end{array}$ & $20^{\prime}$ \\
\hline $\begin{array}{l}\text { Actividad de exposición } \\
\text { de los trabajos }\end{array}$ & $\begin{array}{l}\text { Exposición de los trabajos de los } \\
\text { subgrupos al resto de la clase. }\end{array}$ & $40^{\prime}$ \\
\hline $\begin{array}{l}\text { Actividad de exposición } \\
\text { final del grupo "ideal" }\end{array}$ & $\begin{array}{l}\text { Exposición final del grupo "ideal" } \\
\text { con correcciones y mejoras de } \\
\text { los subgrupos. }\end{array}$ & $20^{\prime}$ \\
\hline $\begin{array}{l}\text { Actividad de reflexión y } \\
\text { debate }\end{array}$ & $\begin{array}{l}\text { Se plantean dudas y resuelven } \\
\text { problemas }\end{array}$ & $15^{\prime}$ \\
\hline $\begin{array}{l}\text { Actividad de cuestionario } \\
\text { final los conocimientos }\end{array}$ & $\begin{array}{l}\text { Recogida de ideas finales } \\
\text { aprendidas por el alumnado }\end{array}$ & $20^{\prime}$ \\
\hline
\end{tabular}

\section{Cuestionarios iniciales y finales}

Cada una de las actividades propuestas para este CIMA se completa con la entrega y realización del alumnado de unos cuestionarios inicial y final empleados para evaluar 
los conocimientos realizados previamente y posteriormente a la actividad y mediante el empleo de escaleras de aprendizaje. Algunas de las mejoras propuestas para este CIMA fue una reducción en el número de preguntas de los cuestionarios así como una reformulación de sus preguntas.

Con respecto al cuestionario sobre los gestores bibliográficos se ha simplificado en algunas preguntas como la 1 , y la 5, y la pregunta 2 se ha reformulado y suprimido en datos para favorecer la claridad en su formulación, la pregunta 3 se ha eliminado por ser redundante en su contenido. En referencia al cuestionario para evaluar los conocimientos sobre las experiencias formativas y salidas profesionales, la pregunta 4 se ha subdivido en una pregunta 5 para darle mayor importancia a la cuestión de género en relación a la profesión y su trato en la relación salud-género. Finalmente el cuestionario sobre la Comunicación y la exposición oral se ha simplificado en su última pregunta.

Se pueden diferenciar unos niveles dentro de la escalera de aprendizaje de cada estudiante y que deben representar su proceso aprendizaje: Se diferencian cuatro grados en función de la complejidad de la respuesta. Grado A: No responde nada o no relacionado con el tema; Grado B: Las respuestas tienen contenidos básicos; Grado C: Conoce y diferencia contenidos; Grado D: Identifica y responde correctamente y con complejidad. También se evaluó la satisfacción global en relación al proceso enseñanza-aprendizaje: Muy poca-poca- suficiente-Bastante-mucha. La evaluación del cuestionario sobre comunicación y la exposición oral, se evaluó mediante el empleo de la herramienta de evaluación Kahoot, como medida de mejora del presente CIMA. Se planteó una evaluación mediante el uso de las TIC a través de Kahoot (Guzman-Duque, 2016) para favorecer el acercamiento digital en los estudiantes, siendo complementaria dado que las 
escaleras de aprendizaje me permiten conocer mejor el proceso y que el empleo de esta herramienta se vió dificultado por cuestiones técnicas. Es una herramienta que no voy a volver a aplicar como acción de mejora en futuros CIMA.

\title{
Cuestionario inicial y final para evaluar los conoci- mientos sobre el gestor bibliográfico.
}

1. ¿Conoces o usas algún gestor de referencias bibliográficas?

2. ¿Qué son y para qué se usan los gestores de referencias?

3. ¿Cómo se organizan y gestionan las referencias?

4. ¿Sabes crear una bibliografia? Pon un ejemplo.

5. ¿Cómo de importante es este taller de aprendizaje para tus estudios de Podología?

6. ¿Consideras que se debe repetir esta actividad? ¿Por qué?

7. ¿Cuáles son los pasos para importar referencias?

\author{
Cuestionario inicial y final para evaluar los conoci- \\ mientos sobre los estilos de Vancouver y APA. \\ 1. ¿Cuáles son los diferentes estilos de referencias \\ bibliográficas? \\ 2. ¿Cuáles son las pautas generales a seguir en los \\ estilos Vancouver? \\ 3. ¿Cuáles son las pautas generales a seguir en los \\ estilos APA? \\ 4. ¿Qué datos bibliográficos se indican en cada uno \\ de los estilos APA y Vancouver?
}

\section{Cuestionario inicial y final para evaluar los cono- cimientos sobre las experiencias formativas y salidas profesionales.}

1. ¿Cuáles son las salidas posgrado de la titulación?

2. ¿Es posible realizar el doctorado? Indica todos los pasos a seguir. 
3. ¿Mi futuro profesional solamente va a ser en una consulta privada?

4. ¿Existen especialidades en Podología?

5. ¿Está masculinizada la profesión de podología? Explícalo.

\section{Cuestionario inicial y final para evaluar los conoci- mientos sobre la Comunicación y la exposición oral.}

1. ¿Para qué sirven los recursos materiales / técnicos en una exposición oral?

2. ¿Cuáles son las características del lenguaje verbal en la comunicación oral?

3. ¿Cuáles son las características del lenguaje no verbal en la comunicación oral?

4. ¿Cuáles son los errores más habituales en la comunicación oral?

5. De manera complementaria se le preguntó al alumnado sobre su satisfacción con los distintos contenidos y proceso de enseñanza-aprendizaje de este CIMA.

\section{Aplicación del CIMA y relato resumido de las sesiones}

La actividad del gestor bibliográfico y la búsqueda de información científica se reforzó con la actividad del taller específico que se realizó son conocer el nivel de informática de los estudiantes y dando por hecho que son nativos digitales. Se formaron 4 subgrupos de clase, integrados por 15 estudiantes. El profesorado estuvo de apoyo al video tutorial para la actividad y finalmente se solicitó una revisión bibliográfica para reforzar el conocimiento adquirido. La mayoría del alumnado presentó interés por el contenido, si bien en algunos casos se observó que existían dificultades en el manejo y falta de interés pero el clima de trabajo fue muy positivo y enriquecedor. En las sesiones de referencias y estilos Vancouver- APA se pretendió 
manejar y conocerlos para su aplicación inmediata en su formación. Se formaron subgrupos de 10 alumnos para el trabajo en grupos reducidos y cada subgrupo se volvió a dividir en grupos de 3 personas para trabajar contenidos más específicos.

El profesorado entregó parte de los contenidos a trabajar y el resto lo elaboraba el grupo mediante búsqueda autónoma de información. De cada subgrupo, se decidió un representante para integrar el grupo definitivo final y que sería el encargado de explicar los contenidos al grupo de clase total. Mi papel fue de coach en todo el proceso y con el asesoramiento para la consecución final de los objetivos planificados mediante el aprendizaje de inteligencia colectiva y trabajo en grupo. En mis notas de campo y diario profesional reflexione sobre las dificultades relacionadas con el manejo de grupo grande y la estructura fisica de las infraestructuras de las clases.

En la preparación y desarrollo de las sesiones de experiencias profesionales y formativas dentro del contenido histórico, legal y profesionalizante de la podología cumple un papel muy importante las emociones. Es el momento de generar emociones y sentimiento y de lanzar un mensaje de "dar una oportunidad". Cada uno de los ponentes emite el mensaje de "...dale una oportunidad...". El video fórum me permite profundizar y reflexionar personalmente en el alumnado y su puesta en común y debate refuerza esa necesidad de motivación y de superación, de conseguir lo que nos proponemos. Y como no, de recompensa personal para mí cuando consigo mis objetivos con su docencia y con la enseñanza. Se realizó un aprendizaje experiencial y de colaboración con personas invitadas y se plantearon los estudios desde el pregrado, hasta el postgrado inmediato y futuro. Analizando contenidos teóricos y prácticos incluyendo la carrera académica y salidas profesionales. La importancia de la formación continuada se refuerza con el carácter sanitario y científico de la profesión. 


\section{Evaluación del aprendizaje de los estudiantes}

La evaluación sobre el aprendizaje de cada una de las actividades de este CIMA se resume en la tabla 5.

Tabla 5. Resultados de los niveles de conocimientos adquiridos en este CIMA.

\begin{tabular}{|c|c|c|c|c|c|c|c|c|c|}
\hline \multirow[b]{3}{*}{ Cuestionario } & \multirow[b]{3}{*}{ Preguntas } & \multicolumn{8}{|c|}{ Niveles de conocimientos } \\
\hline & & \multicolumn{2}{|c|}{ Nivel A } & \multicolumn{2}{|c|}{ Nivel B } & \multicolumn{2}{|c|}{ Nivel C } & \multicolumn{2}{|c|}{ Nivel D } \\
\hline & & Pre & Post & Pre & Post & Pre & Post & Pre & Post \\
\hline \multirow[t]{7}{*}{1} & 1 & $81,0 \%$ & $15,0 \%$ & $18,0 \%$ & $76,0 \%$ & $1,0 \%$ & $9,0 \%$ & $0,0 \%$ & $0,0 \%$ \\
\hline & 2 & $79,0 \%$ & $21,0 \%$ & $20,0 \%$ & $64,0 \%$ & $1,0 \%$ & $15,0 \%$ & $0,0 \%$ & $0,0 \%$ \\
\hline & 3 & $94,0 \%$ & $32,0 \%$ & $6,0 \%$ & $52,0 \%$ & $0,0 \%$ & $16,0 \%$ & $0,0 \%$ & $0,0 \%$ \\
\hline & 4 & $92,0 \%$ & $41,0 \%$ & $8,0 \%$ & $47,0 \%$ & $0,0 \%$ & $12,0 \%$ & $0,0 \%$ & $0,0 \%$ \\
\hline & 5 & $74,0 \%$ & $29,0 \%$ & $26,0 \%$ & $62,0 \%$ & $0,0 \%$ & $9,0 \%$ & $0,0 \%$ & $0,0 \%$ \\
\hline & 6 & $75,0 \%$ & $46,0 \%$ & $16,0 \%$ & $42,0 \%$ & $9,0 \%$ & $12,0 \%$ & $0,0 \%$ & $0,0 \%$ \\
\hline & 7 & $92,0 \%$ & $33,0 \%$ & $8,0 \%$ & $60,0 \%$ & $0,0 \%$ & $7,0 \%$ & $0,0 \%$ & $0,0 \%$ \\
\hline \multirow[t]{4}{*}{2} & 1 & $48,0 \%$ & $6,0 \%$ & $49,0 \%$ & $5,0 \%$ & $3,0 \%$ & $9,0 \%$ & $0,0 \%$ & $80,0 \%$ \\
\hline & 2 & $86,0 \%$ & $11,0 \%$ & $12,0 \%$ & $27,0 \%$ & $2,0 \%$ & $62,0 \%$ & $0,0 \%$ & $0,0 \%$ \\
\hline & 3 & $92,0 \%$ & $14,0 \%$ & $6,0 \%$ & $41,0 \%$ & $2,0 \%$ & $45,0 \%$ &, $0 \%$ & $0,0 \%$ \\
\hline & 4 & $87,0 \%$ & $16,0 \%$ & $13,0 \%$ & $69,0 \%$ & $0,0 \%$ & $15,0 \%$ & $0,0 \%$ & $0,0 \%$ \\
\hline \multirow[t]{5}{*}{3} & 1 & $39,0 \%$ & $6,0 \%$ & $42,0 \%$ & $24,0 \%$ & $18,0 \%$ & $42,0 \%$ & $0,0 \%$ & $28,0 \%$ \\
\hline & 2 & $72,0 \%$ & $7,0 \%$ & $22,0 \%$ & $19,0 \%$ & $6,0 \%$ & $8,0 \%$ & $0,0 \%$ & $66,0 \%$ \\
\hline & 3 & $36,0 \%$ & $17,0 \%$ & $38,0 \%$ & $15,0 \%$ & $26,0 \%$ & $40,0 \%$ & $0,0 \%$ & $28,0 \%$ \\
\hline & 4 & $24,0 \%$ & $12,0 \%$ & $72,0 \%$ & $52,0 \%$ & $4,0 \%$ & $28,0 \%$ & $0,0 \%$ & $8,0 \%$ \\
\hline & 5 & $61,0 \%$ & $29,0 \%$ & $27,0 \%$ & $46,0 \%$ & $12,0 \%$ & $23,0 \%$ & $0,0 \%$ & $2,0 \%$ \\
\hline \multirow[t]{4}{*}{4} & 1 & $64,0 \%$ & $17,0 \%$ & $36,0 \%$ & $62,0 \%$ & $0,0 \%$ & $19,0 \%$ & $0,0 \%$ & $2,0 \%$ \\
\hline & 2 & $44,0 \%$ & $8,0 \%$ & $64,0 \%$ & $23,0 \%$ & $2,0 \%$ & $57,0 \%$ & $0,0 \%$ & $12,0 \%$ \\
\hline & 3 & $51,0 \%$ & $10,0 \%$ & $47,0 \%$ & $61,0 \%$ & $2,0 \%$ & $13,0 \%$ & $0,0 \%$ & $16,0 \%$ \\
\hline & 4 & $59,0 \%$ & $12,0 \%$ & $38,0 \%$ & $63,0 \%$ & $3,0 \%$ & $16,0 \%$ & $0,0 \%$ & $9,0 \%$ \\
\hline
\end{tabular}

La evaluación del aprendizaje del alumno mediante el uso de las escaleras de aprendizaje permite conocer la 
evolución de las ideas de los estudiantes. Los resultados muestran la mejora en el aprendizaje en el grupo. Algunas de los resultados más destacados se pueden observar en las siguientes escaleras de aprendizaje de este CIMA (Figura 5,6,7,8). La satisfacción global final con las actividades de este proceso de enseñanza y aprendizaje y los distintos contenidos fueron de Búsqueda de información bibliográfica y Gestor de referencias bibliográficas Mendeley (Poca: 18\%; Suficiente: 40\%; Bastante: 19\%; Mucha: 23\%) Exposición Oral. ¿Cómo hablar en público? (Poca: 12\%; Suficiente: 23\%; Bastante: 32\%; Mucha: 33\%). Futuras experiencias profesionales (Suficiente: 15\%; Bastante: 11\%; Mucha: 74\%) Estilos Vancouver-APA (Poca: 8\%; Suficiente: 29\%; Bastante: 49\%; Mucha: $14 \%$ ) y en general, cuál ha sido su satisfacción con el proceso de enseñanza-aprendizaje (Suficiente: 7\%; Bastante: 21\%; Mucha: 72\%).

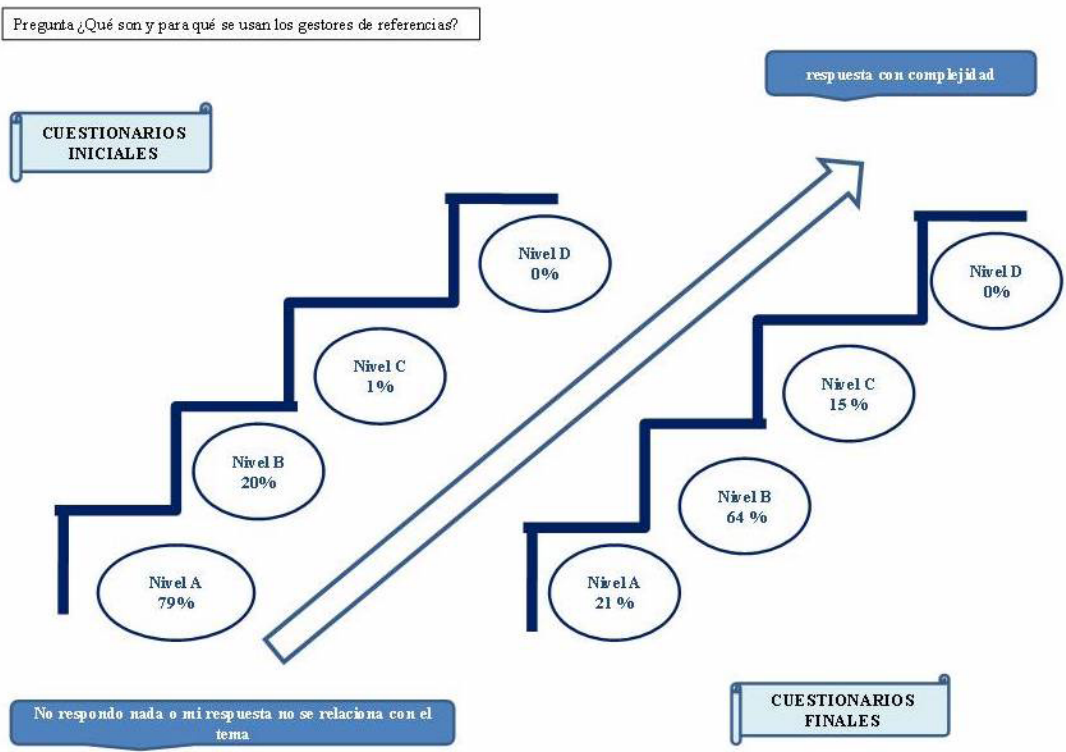

Figura 5. Escalera de aprendizaje de la pregunta 2 sobre los gestores bibliográficos.

Jornadas de Formación e Innovación Docente del Profesorado | № 2 (2019) Esta obra se distribuye con la licencia Creative Commons Reconocimiento-NoComercial-SinObraDerivada 


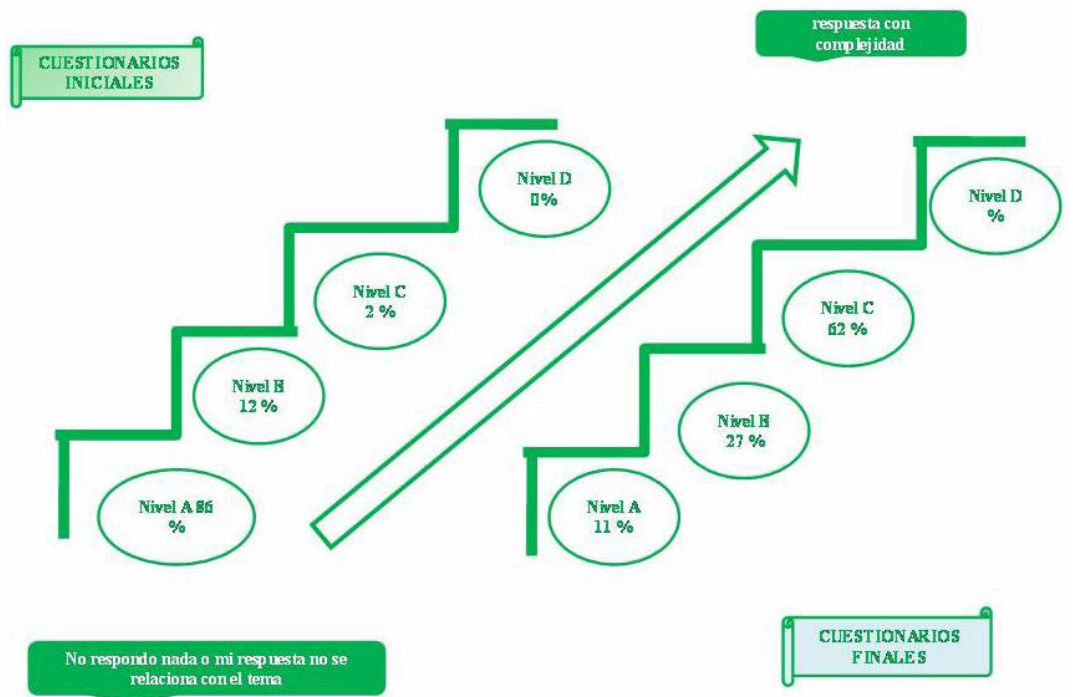

Figura 6. Escalera de aprendizaje de la pregunta 2 sobre los estilos vancouver.

Pregunta $i$ Es posible realizar d doctorado? Indica todos los pasos a seguir.

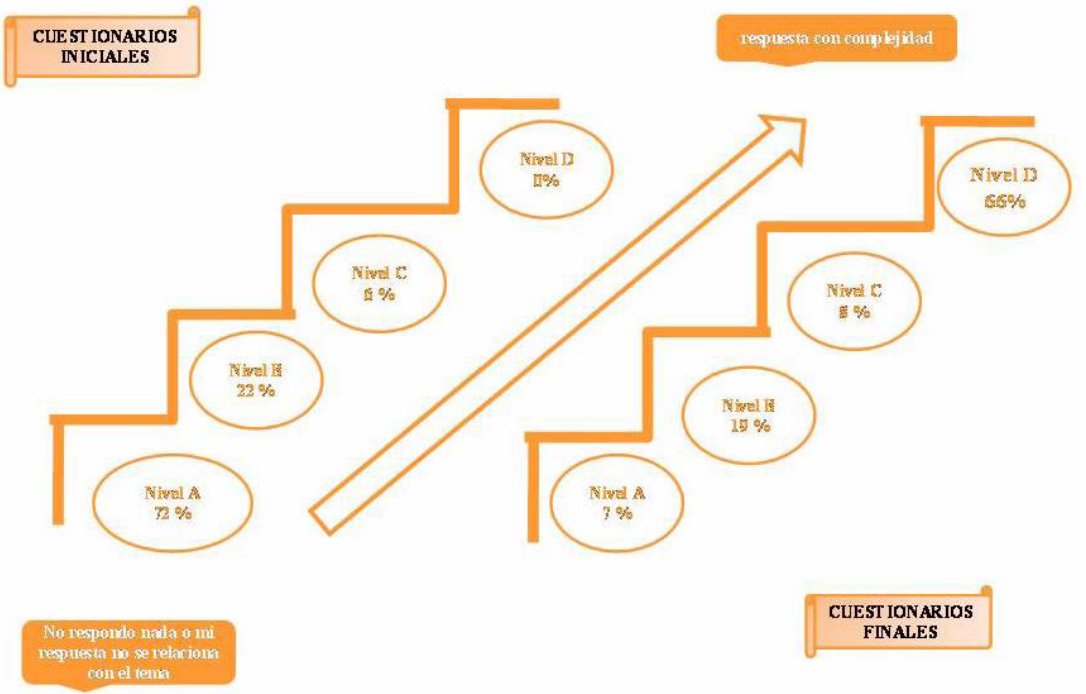

Figura 7. Escalera de aprendizaje de la pregunta 2 sobre el desarrollo profesional.

Jornadas de Formación e Innovación Docente del Profesorado | № 2 (2019) 
Figura 8. Escalera de aprendizaje de la pregunta 4 sobre la comunicación.

\section{Evaluación del CIMA y principios didácticos}

La motivación y el interés por los contenidos de este CIMA en los estudiantes aumentan la capacidad de aprendizaje de éstos, y ha favorecido la obtención de estos resultados de los cuestionarios finales de evaluación. Se ha hecho uso del diario de profesor, como diario profesional, para poder analizar y describir los aspectos más importantes en este CIMA y gracias a él, me ha permitido recoger impresiones que han facilitado realizar una reflexión sobre mi docencia. Durante unos minutos a la semana se ha escrito y reflexionado sobre lo ocurrido en las clase y me han permitido tomar conciencia como docente del desarrollo del CIMA y de aspectos a mejorar o que han sido de gran utilidad. La gestión del tiempo me ha permitido concluir que necesito dedicar más tiempo al debate y la discusión de los estudiantes para facilitar la creación de sinergias positivas entre ellos. La experiencia previa me confirma que en cuanto a los cuestionarios he detectado que son 
recibidos de manera muy positiva por el alumnado. Los estudiantes cumplimentaron un cuestionario anónimo de evaluación de la docencia, para ello emplearon el nombre de una ciudad que coincidía con la primera inicial de su apellido. Este hecho ha generado cierta confusión en algunos de ellos y que yo interpreto como de inseguridad, pues en algunos de los cuestionarios el alumnado ponía los dos nombres, el suyo y el de su ciudad o nombre anónimo. Esto lo interpreto como un refuerzo de su personalidad, pero no estoy seguro. Mediante el análisis cualitativo de estos cuestionarios he podido localizar fortalezas y debilidades en relación al proceso de aprendizaje y enseñanza con el alumnado.

Con respecto a mis emociones he de reconocer que pese a que se repite la innovación metodológica con algunas mejoras, durante el inicio de la misma el nerviosismo y la preocupación por que salga todo bien, se han apoderado de mí. Pero rápidamente, estos sentimientos se han convertido en positivos y han sido productivos en el desarrollo de este ciclo de mejora en el aula. En otras ocasiones he detectado que una parte del alumnado se sorprendía y no eran conscientes del rol activo que desempeñaban en este ciclo de mejora, en este proceso de aprendizaje, llegando incluso a cuestionarme la educación en la transmisión del conocimiento. Algunos alumnos echaron en falta más clases y contenidos teóricos. En otras ocasiones este papel más activo era desempeñado por estudiantes mediante el azar, de manera que esa aleatorización generaba sorpresa en ellos mismos.

Destaco de manera muy importante y sorpresiva para mí, la creación de la figura de "persona sociable" por el alumnado durante el desarrollo de los subgrupos de la actividad de comunicación y exposición oral en público. Esta denominación para la persona integrante del grupo ideal fue algo que surgió de varios subgrupos como si estuviese programado. Esto me hace pensar que es la denominación adecuada y que más entiende el grupo y que por tanto la voy a integrar en este CIMA. 
La satisfacción con la dinámica participativa en las clases fue unánime. Los estudiantes valoraron positivamente la realización de este CIMA y el uso de medios audiovisuales para generar sesiones más dinámicas. El trabajo de actualización y análisis realizado con los mapas de contenidos junto con el alumnado ha permitido enriquecerlos y favorecer la compresión y el aprendizaje de los contenidos trabajados. Los estudiantes reconocieron que las actividades desarrolladas fueron dinámicas y atractivas para el aprendizaje, lo que refuerza la efectividad de la innovación metodológica empleada en este CIMA. Como planteamiento futuro pretendo continuar con este CIMA por ser una metodología de innovación necesaria y útil cuyos resultados me indican que son eficaces para el aprendizaje de la asignatura de fundamentos de podología. El carácter dinámico de los mapas de contenidos me motiva a mí y a los estudiantes y se irá adaptando a cada curso en su aplicación.

Con respecto al sistema de evaluación pese a ser una labor muy tediosa y que requiere mucho tiempo y de dedicación por el profesor considero que es la que mayor información me aporta sobre el nivel de conocimientos que adquiere el alumnado. Por otra parte, el empleo de Kahoot para la futura aplicación lo descarto por no ser adecuado ni de utilidad para mi evaluación. El resto del CIMA considero que se está consolidando con el paso de las experiencias pasadas. La entrega de este artículo en tampoco tiempo no es adecuada al proceso por su premura en el tiempo. Algunos de los principios didácticos argumentados que deben permanecer en el futuro son (Porlán et al. 2017) el diseño de cuestionario de ideas previas sobre el conocimiento del alumnado, la elaboración de mapas de contenidos para ordenar y jerarquizar los contenidos con el uso de una pregunta intrigante y la realización de "diario del profesor" para la reflexión diaria del desarrollo de las sesiones. 


\section{Referencias bibliográficas}

Amador, L., Monreal, M.C. y Marco, M.J. (2001). El adulto: etapas y consideraciones para el aprendizaje. Eúphoros, 3 , 97-112.De Alba, N. y Porlán, R. (2017). La metodología de enseñanza. En R. Porlán (Coord.), Enseñanza universitaria. Cómo mejorarla (37-53). Madrid: Ediciones Morata.

Coheña Jiménez, M. (2018). Ciclo de mejora de fundamentos de Podología. Jornadas de Formación e Innovación Docente del Profesorado, 1, 996-1015.

Finkel, D. (2000). Teachingwithyourmouthshut. Portsmouth, NH. HeinemannBoynton/Cook. (Trad. Ast.: Dar clase con la boca cerrada. Valencia. Publicaciones de la Universidad de Valencia, 2008).

García, E., Porlán, R. y Navarro, E. (2017). Los fines y los contenidos. En R. Porlán (Coord.), Enseñanza universitaria. Cómo mejorarla (55-72). Madrid: Ediciones Morata.

Guerra-Martín, MD., Lima-Serrano, M., Zambrano-Domínguez, EM. y Fernández-Rodríguez, V. (2013). Evaluacioón de una intervención sobre busquedas de información científica para estudiantes de enfermería. Texto \& Contexto- Enfermagem, 22: 619-628.

Guerra-Martin, M D, Lima-Serrano, M., Zambrano-Domínguez, EM. y Lima-Rodríguez, JS. (2014) ¿ES efectivo el aprendizaje sobre alfabetización en información para estudiantes de enfermería? Enfermería Global, 13, 90-102.

Guzmán-Duque, A., Mendoza-Paredes, J., y. Tavera-Castillo, N. (2016). Kahoot!: un mecanismo de innovación para la educación universitaria. En R. Roig- vila, (Coord). El compromiso académico y social de la investigación e innovación educativa en la enseñanza superior. (633640). Barcelona: Editores Octaedro. 